\title{
Iterative homology checking and non-uniform stepping during RecA-mediated strand exchange
}

Yu-Wei Zhang ${ }^{\mathrm{a}}$, Da-Guan Nong ${ }^{\mathrm{a}}$, Shuo-Xing Dou ${ }^{\mathrm{a}}$, Wei Li ${ }^{\mathrm{a}}$, Yan Yan ${ }^{\mathrm{a}, \mathrm{d}}, \mathrm{Xu}-\mathrm{Guang} \mathrm{Xi}^{\mathrm{b}, \mathrm{c}}$, Chun-Hua Xu ${ }^{\mathrm{a}, *} \&$ Ming Li ${ }^{\mathrm{a}, *}$

${ }^{a}$ Beijing National Laboratory for Condensed Matter Physics and Key Laboratory of Soft Matter Physics, Institute of Physics, Chinese Academy of Sciences, Beijing 100190, China

${ }^{\mathrm{b}}$ College of Life Sciences, Northwest A \& F University, Xi'an, Shaanxi 712100, China

${ }^{c}$ LBPA, ENS de Cachan, CNRS, Université Paris-Saclay, F-94235 Cachan, France

${ }^{\mathrm{d}}$ Department of Physics, Emory University, Atlanta, GA 30322, USA

*Correspondence: mingli@ iphy.ac.cn (M.L.), xch@iphy.ac.cn (C.H.X.) 


\section{Abstract}

Recombinase-mediated homologous recombination (HR) in which strands are exchanged between two similar or identical DNA molecules is essential for maintaining genome fidelity and generating genetic diversity. It is believed that HR comprises two distinct stages: an initial alignment with stringent homology checking followed by stepwise heteroduplex expansion. If and how homology checking takes place during heteroduplex expansion, however, remains unknown. In addition, the number of base pairs (bp) involved in each step is still under debate. By using single-molecule approaches to catch transient intermediates in RecA-mediated HR with different degrees of homology, we show that $(i)$ the expansion proceeds with step sizes of multiples of $3 \mathrm{bp}$, (ii) the step sizes follow wide distributions that are similar to that of initial alignment lengths, and (iii) each distribution can be divided into a short-scale and a long-scale part irrespective of the degree of homology. Our results suggest an iterative mechanism of strand exchange in which ssDNA-RecA filament interrogates double-stranded DNA using a short tract (6-15 bp) for quick checking and a long tract (>18 bp) for stringent sequence comparison. The present work provides novel insights into the physical and structural bases of DNA recombination.

Keywords: Homologous recombination; RecA; Homology recognition; Heteroduplex expansion; Single-molecule techniques

\section{Introduction}

As a prototypical member of recombinases, RecA from Escherichia coli has been extensively studied[1,2]. RecA monomers polymerize along a single-stranded DNA 
(ssDNA) to form a helical filament with $\sim 6$ monomers and $\sim 18$ nucleotides per turn[3,4,5,6]. The ssDNA-RecA filament searches for homology in a double-stranded DNA (dsDNA) template and invades the template to produce a heteroduplex that expands to complete the homologous recombination (HR)[7]. The minimum length required for efficient initial alignment for sequence checking has been disputed for a long time. It was reported to be as small as $6-14 \mathrm{bp}[8,9,10,11,12,13,14,15]$ or as large as $20-30 \mathrm{bp}[16,17]$. Recently, the issue appeared to have been settled by single-molecule assays which showed that HR might involve an extremely stringent initial checking of $\sim 8$ contiguous bases[9,11,15]. But the connection between the alignment length and the structural feature of ssDNA-RecA filament is still elusive. As to the expansion, it is generally believed that the heteroduplex expands in 3-bp steps (Fig. 1A)[11,14,18], which seemed to be logical because a base triplet is the natural length unit of a ssDNA-RecA filament[6,18]. However, the triplet-by-triplet expansion mechanism would suggest that, due to the energy cost of base-pair breaking, even a single mismatch in front of the heteroduplex might strongly hinder or even block the strand-exchange process. It therefore cannot explain why HR may tolerate a relatively high degree of mismatch[12,19,20].

\section{Material and methods}

\subsection{DNA construction for FRET assay}

All oligonucleotides required to make the DNA substrates were purchased from Sangon Biotech (Shanghai, China). The oligonucleotides were suspended in TE buffer (10 mM Tris-HCl, $1 \mathrm{mM}$ EDTA, $\mathrm{pH}$ 8.0). The dsDNA molecules were prepared by mixing complementary DNA molecules and heating to $95^{\circ} \mathrm{C}$ followed by slow cooling to 
room temperature over a period of 6 hours. A $2 \%$ agarose gel was used to purify the dsDNA to ensure the absence of free ssDNA in the prepared DNA. All oligonucleotides for the FRET assay are listed in Supplementary Table T1.

\subsection{DNA construction for Magnetic Tweezers(MT) assay}

The DNA substrate used in the MT assay is sketched in Supplementary Figs. S1, where the olive segment is complementary to the RecA-coated ssDNA. The 3-way DNA structure was prepared by mixing oligos $\mathrm{H} 1, \mathrm{H} 2$, Stem 1 and Stem 2 together and heating to $95^{\circ} \mathrm{C}$ followed by slow cooling to room temperature over a period of 6 hours. The dsDNA segment was elongated by adding a 190-bp dsDNA whose right end was sealed by Seal1 and Seal2. The resulting dsDNA template is 270-bp long. The two stems were connected to two 750-bp dsDNA handles. The ends of the two handles were labeled with biotin and digoxigenin respectively. All the oligonucleotides for MT assays are listed in

\section{Supplementary Table T2.}

\subsection{FRET assay}

The single-molecule FRET measurements were carried out with a home-built objective-type TIRF microscopy. An oil immersion objective (100x, N.A.1.49) was used to generate the evanescent field of illumination. Cy3 is excited by a $532 \mathrm{~nm}$ Sapphire laser (Coherent Inc., U.S.). The fluorescence signals from Cy3 and Cy5 were split by a dichroic mirror, and collected by an EMCCD (iXON, Andor Technology, South Windsor, CT). The coverslips (Fisher Scientific, U.S.) and slides were cleaned thoroughly by a mixture of sulfuric acid and hydrogen peroxide with a volume ratio of $7: 3$, acetone and 
sodium ethoxide. The coverslips were coated with a mixture of $99 \%$ of mPEG (m-PEG-5000, Laysan Bio, Inc.) and 1\% of biotin-PEG (biotin-PEG-5000, Laysan Bio, Inc.).

The donor-labeled partial duplex DNA molecules were immobilized on the passivated surface by means of a biotin-streptavidin interaction. After washing away the excess DNA molecules, the partial duplex DNA substrates were incubated with $1 \mu \mathrm{M}$ RecA (New England Biolabs) and $1 \mathrm{mM} \mathrm{ATP} \gamma \mathrm{S}$ (Roche Diagnostics) in the RecA reaction buffer for $15 \mathrm{~min}$ to ensure formation of the ssDNA-RecA filaments. In the assays with mismatched dsDNA, $10 \mathrm{nM}$ dsDNA and $1 \mathrm{mM}$ ATP $\gamma \mathrm{S}$ was incubated with the ssDNA-RecA filaments for 5 min to ensure maximized binding of the dsDNA. Imaging was initiated after washing away the excess dsDNA with the RecA reaction buffer containing $1 \mathrm{mM}$ ATP $\gamma \mathrm{S}$ and an oxygen scavenging system $(1 \mathrm{mg} / \mathrm{ml}$ glucose oxidase, $0.8 \%$ glucose, $0.4 \mathrm{mg} / \mathrm{ml}$ catalase and $1 \mathrm{mM}$ Trolox). However, for the assay of completely homologous dsDNA, after the formation of ssDNA-RecA filaments, the buffer in the chamber was exchanged with a solution of $500 \mathrm{pM}$ dsDNA and $1 \mathrm{mM}$ $\mathrm{ATP} \gamma \mathrm{S}$ in the RecA reaction buffer supplemented with the oxygen scavenging system. Imaging was initiated as soon as the buffer exchange was complete.

\subsection{MT assay}

A flow chamber was assembled with a glass slide and a cover slide, and was placed on an inverted microscope (IX71, Olympus). Both slides were cleaned and the cover slide was modified with anti-digoxigenin proteins. A magnetic bead was tethered to the modified cover slide through a single DNA substrate. An external magnetic force was 
applied on the magnetic bead by placing a permanent magnet above the chamber. The distance between the magnetic bead and the cover slide, that is, the DNA extension, was monitored by analyzing the shape of the diffraction rings of the magnetic bead which depends on the distance between the bead and the focal plane of the objective (100x, NA 1.45 , Olympus).

$150 \mathrm{nM}$ RecA (New England Biolabs), $5 \mathrm{nM}$ ssDNA and $1 \mathrm{mM}$ ATP $\gamma \mathrm{S}$ (Roche Diagnostics) were pre-incubated in the RecA reaction buffer containing $70 \mathrm{mM}$ Tris- $\mathrm{HCl}$ (pH 7.5), $10 \mathrm{mM} \mathrm{MgCl}$ and $5 \mathrm{mM}$ dithiothreitol (DTT) at $37^{\circ} \mathrm{C}$ for $15 \mathrm{~min}$ to form ssDNA-RecA filaments. The ssDNA-RecA filaments were diluted to $2.5 \mathrm{nM}$ (in DNA molecules). After checking the state of the DNA-magnetic bead connection, the solution was introduced into the chamber. The HR process was monitored by recording the magnetic bead-surface distance as a function of time.

\section{Results}

\subsection{FRET measurement of the heteroduplex expansion}

Previous studies have indicated that, after the initial sequence recognition, strand exchange proceeds with a characteristic rate of $2-6 \mathrm{bp} / \mathrm{s}[21,22,23]$. Such a modest process should be readily detectable in a single-molecule FRET assay (Fig. 1B)[14]. When the dsDNA template is incorporated into the RecA filament, the average base-to-base distance will be stretched to $0.51 \mathrm{~nm}, 50 \%$ longer than that of B-DNA. In our approach, the labeling sites for the dye molecules (Cy3 and Cy5) were so positioned that the FRET efficiency would change significantly when the last $21 \mathrm{bp}$ of the dsDNA 
template are incorporated into the filament (Fig. 1C). We hence anticipated that it would take $>3.5 \mathrm{~s}$ for the strand-exchange process to complete. Unexpectedly, we observed the FRET efficiency did not increase uniformly with a finite rate. Instead, it jumped abruptly from an initial value to $\sim 1.0$, indicating that the heteroduplex expands with steps much larger than 3 bp (Fig. 1D-F and Supplementary Figs. S2). In addition, there are sometimes short pauses within big jumps. Our results hence are challenging the strand-exchange model in Fig. 1A[11,14,18].

\subsection{Stepwise heteroduplex expansion measured by MT}

A disadvantage of the FRET assay is that only a very small part of the strand-exchange process can be monitored due to its limited probing distance $(<10 \mathrm{~nm})$. We thus designed a magnetic tweezers assay to study the process in more details (Fig. 2A). While $\sim 14 \mathrm{pN}$ is required to pull open a DNA duplex, a much weaker force is enough to open the dsDNA template when strand exchange occurs. The data of strand-exchange with no mismatch were acquired at $\sim 7 \mathrm{pN}$ in order to suppress thermal fluctuations(Supplementary Figs. S3). Here again, strand-exchange was found to progress in big steps (Fig. 2B and Supplementary Figs. S5A), with a size distribution centered at 21 bp (Supplementary Figs. S5B). Close examination indicates that many of the big steps are composed of 2-3 small steps (inset, Fig. 2B). When the small steps are taken into account, the distribution of strand-exchange steps exhibits several sharp sub-maxima at multiples of the base triplet (Fig. 2C). The initial steps in the traces were not included, ensuring that the distribution was solely for heteroduplex expansion (see 
below for further discussion).

3.3. Strand-exchange process at different degrees of mismatch

Using the same MT setup, we also measured the dynamics of strand exchange with dsDNA templates that contain mismatches. When the degree of mismatch increased from $10 \%$ to $20 \%$, the proportion of completed strand-exchange events was reduced from $\sim 40 \%$ to $<10 \%$. In addition, when the mismatches exceeded $20 \%$, the strand-exchange proceeded with some reverses (Fig. 2D) or even could not be completed (Supplementary Figs. S7). These results are consistent with a model in which the ssDNA-RecA filament checks for homology before each step of heteroduplex expansion. To further test the model, we re-designed the FRET experiment to monitor the expansion process directly (Fig. 3A). It is similar to that in Fig. 1A except that the first $30 \mathrm{bp}$ of the dsDNA template are homologous to the first 30 nt of the RecA-coated ssDNA, while the rest are partially homologous. Fig. 3B and Fig. 3C show representative FRET patterns for two high degrees of mismatch, 52\% and 74\%, respectively. Although the strand-exchange process could not complete when the degree of mismatch was high, it was not halted, but rather, the dsDNA was elongated and then restored to its original length repetitively, suggesting that the sequence of the dsDNA was being checked.

3.4. The initiation of strand-exchange measured by MT

By design, the FRET assay in Fig. 3A mimics an actual heteroduplex expansion 
process because a 30-bp heteroduplex has already formed prior to data acquisition. To observe just the initial checking, we next performed an MT assay using a poly(T)-RecA filament (equivalent to $79 \%$ mismatches). In this case, the strand-exchange process did not proceed beyond the initial checking step (Fig. 4A). The distribution of initial checking (or alignment) lengths (Fig. 4B) is similar to that of the expansion step sizes in Fig. 2C. This similarity motivated us to analyze the distribution of the first steps in the previous MT assays without mismatches, which should represent the statistics of initial alignment lengths. Clearly, this distribution (Fig. 4C) and that of expansion step sizes (Fig. 2C) are very similar. This implies that the reactions during the initial checking and the heteroduplex expansion are strongly correlated, or more concretely, sequence checking should be performed in each step of heteroduplex expansion.

\section{Discussion}

All the distributions from our MT assays, for both initial alignment lengths and expansion step sizes, no matter how high the mismatches are, have two global maxima that are centered at 12 and 21 bp, respectively (Figs. 4B, 4C and 2C). In accordance with this, the distributions of FRET efficiencies also have two global maxima, centered at 0.68 (corresponding to $9 \mathrm{bp}$ ) and 0.91 (corresponding to $18 \mathrm{bp}$ ) (Figs. 3D, 3E), respectively. A reasonable explanation of the 3-bp discrepancy between the two assays is the spatial separation $(\sim 2.5 \mathrm{~nm})$ between the primary binding site (PBS) and the secondary binding site (SBS) of RecA for DNA (Fig. 3A)[6]. When two strands of the dsDNA template are unpaired and bind to both sites simultaneously, 3-4 bases at the junction between the pairing region and the intact template are left unpaired according to De Vlaminck et al.[9]. These unpaired bases do not contribute to the elongation of dsDNA template in the FRET 
assay. However, they do make unwanted contribution to the measured ssDNA length changes in the MT assay because they can be readily separated by the external force. It is interesting to notice that, after the 3-bp correction for the MT data, the short-scale global maximum corresponds to a length that is just half of a filament period ( $9 \mathrm{bp}$ ), whereas the long-scale one corresponds to a length that just equals a filament period (18 bp). This clearly suggests that the filament structure plays a critical role in determining the scales of strand-exchange reactions. Consistently, the short-scale parts of the length distributions around 9 bp (colored purple, Figs. 2C, 4B, 4C) remain similar at different homologies.

Note that the relative proportion of the long-scale parts (colored yellow) increases with increasing homology. It suggests that the tendency of a short-scale reaction complex proceeding forward (to form a long complex) or backward (to disintegrate) is influenced by the degree of homology: the higher the homology, the higher the probability for the short-scale complex to grow long. The influence is, however, not so strong because the proportion of the long-scale part is not low even when the degree of mismatches is very high ( $\geq 74 \%$ ) (Figs. 3E and 4B). Nevertheless, when the length of a reaction complex accumulates (after 2-3 reaction sub-steps) to beyond one filament period, the sequence may be checked more stringently because more than $90 \%$ of the strand-exchange events failed to progress longer than $21 \mathrm{bp}$ even when the degree of mismatch is $20 \%$ only (Supplementary Figs. S7).

Strong evidence has been given that the free energy landscape of strand-exchange intermediates exhibits a series of descending steps, with the incorporation of a base triplet into the ssDNA-RecA filament lowering the free energy by $\sim 0.3 \mathrm{~K}_{\mathrm{B}} \mathrm{T}[11,18]$ (Fig. $4 \mathrm{D}$ ). Our results are not contradictory to this because the strand-exchange process needs not to 
follow strictly the smallest descending steps in the free energy landscape (Pathway 1).

Instead, it may stride over several small steps in a single sequence-checking reaction

(Pathway 2). Our results support a mechanism that the strand-exchange proceeds with

"checking and expansion" cycles iteratively until the HR process completes or encounters

a highly mismatched segment. An advantage of this mechanism is that the strand-exchange process can tolerate a finite degree of mismatch while checking a relatively long segment. Otherwise, if the heteroduplex expansion only occurs triplet-by-triplet, a few adjacent mismatches would block the whole reaction, no matter how high the average degree of homology is.

Acknowledgements This work was supported by the National Science Foundation of China [grant numbers. 61275192, 11574382 and 11574381].

\section{References:}

[1] M.M. Cox, Motoring along with the bacterial RecA protein, Nature Rev. Mol. Cell Biol. 8 (2007) 127-138.

[2] S.C. Kowalczykowski, Structural biology: Snapshots of DNA repair, Nature 453 (2008) 463-466.

[3] E. Dicapua, A. Engel, A. Stasiak, T. Koller, Characterization of Complexes between Reca Protein and Duplex DNA by Electron-Microscopy, J. Mol. Biol. 157 (1982) 87-103.

[4] D.F. Dombroski, D.G. Scraba, R.D. Bradley, A.R. Morgan, Studies of the Interaction of Reca Protein with DNA, Nucleic Acids Res. 11 (1983) 7487-7504.

[5] V.A. Lantsov, Homologous DNA transferase RecA: functional activities and the search for homology by recombining DNA molecules, Molekuliarnaia Biologiia 41 (2007) 467-477.

[6] Z. Chen, H. Yang, N.P. Pavletich, Mechanism of homologous recombination from the RecA-ssDNA/dsDNA structures, Nature 453 (2008) 489-484.

[7] M.M. Cox, I.R. Lehman, Reca Protein of Escherichia-Coli Promotes Branch Migration, a Kinetically Distinct Phase of DNA Strand Exchange, Proc. Nati. Acad. Sci. 78 (1981) 3433-3437.

[8] A. Bucka, A. Stasiak, RecA-mediated strand exchange traverses substitutional heterologies more easily than deletions or insertions, Nucleic Acids Res. 29 (2001) 2464-2470.

[9] I. De Vlaminck, M.T. van Loenhout, L. Zweifel, J. den Blanken, K. Hooning, S. Hage, J. Kerssemakers, C. Dekker, Mechanism of homology recognition in DNA recombination from dual-molecule experiments, Mol. Cell 46 (2012) 616-624.

[10] J. Xiao, A.M. Lee, S.F. Singleton, Direct evaluation of a kinetic model for RecA-mediated DNA-strand exchange: the importance of nucleic acid dynamics and entropy during homologous genetic recombination, ChemBioChem 7 (2006) 1265-1278.

[11] Z. Qi, S. Redding, J.Y. Lee, B. Gibb, Y. Kwon, H. Niu, W.A. Gaines, P. Sung, E.C. Greene, DNA sequence alignment by microhomology sampling during homologous recombination, Cell 160 (2015) 856-869.

[12] C. Danilowicz, D. Yang, C. Kelley, C. Prevost, M. Prentiss, The poor homology stringency in the 
heteroduplex allows strand exchange to incorporate desirable mismatches without sacrificing recognition in vivo, Nucleic Acids Res. 43 (2015) 6473-6485.

[13] R. Sharma, A.G. Davies, C. Wälti, Directed assembly of 3-nm-long RecA nucleoprotein filaments on double-stranded DNA with nanometer resolution, ACS nano 8 (2014) 3322-3330.

[14] K. Ragunathan, C. Joo, T. Ha, Real-time observation of strand exchange reaction with high spatiotemporal resolution, Structure 19 (2011) 1064-1073.

[15] P. Hsieh, C.S. Cameriniotero, R.D. Cameriniotero, The synapsis event in the homologous pairing of DNAs: RecA recognizes and pairs less than one helical repeat of DNA, Proc. Nati. Acad. Sci. 89 (1992) 6492-6496.

[16] D. Sagi, T. Tlusty, J. Stavans, High fidelity of RecA-catalyzed recombination: a watchdog of genetic diversity, Nucleic Acids Res. 34 (2006) 5021-5031.

[17] P. Shen, H.V. Huang, Homologous Recombination in Escherichia-Coli - Dependence on Substrate Length and Homology, Genetics 112 (1986) 441-457.

[18] J.Y. Lee, T. Terakawa, Z. Qi, J.B. Steinfeld, S. Redding, Y. Kwon, W.A. Gaines, W. Zhao, P. Sung, E.C. Greene, Base triplet stepping by the Rad51/RecA family of recombinases, Science 349 (2015) 977-981.

[19] M.E. Bianchi, C.M. Radding, Insertions, deletions and mismatches in heteroduplex DNA made by recA protein, Cell 35 (1983) 511-520.

[20] A.S. Patrice Morel, S. Dusko Ehrlich, and Era Cassuto, Effect of Length and Location of Heterologous Sequences on RecA-mediated Strand Exchang, J. Biol. Chem. 269 (1994) 19830-19835.

[21] W.A. Bedale, M. Cox, Evidence for the coupling of ATP hydrolysis to the final (extension) phase of RecA protein-mediated DNA strand exchange, J. Biol. Chem. 271 (1996) 5725-5732.

[22] T. van der Heijden, M. Modesti, S. Hage, R. Kanaar, C. Wyman, C. Dekker, Homologous recombination in real time: DNA strand exchange by RecA, Mol. Cell 30 (2008) 530-538.

[23] A. Peacock-Villada, D. Yang, C. Danilowicz, E. Feinstein, N. Pollock, S. McShan, V. Coljee, M. Prentiss, Complementary strand relocation may play vital roles in RecA-based homology recognition, Nucleic Acids Res. 40 (2012) 10441-10451. 


\section{Figure Legends}

Figure 1 (color in print) FRET-based strand-exchange assay with complete homology. (A) Schematic diagram showing the widely recognized mechanism in which the heteroduplex expands precisely in 3-bp steps. RecA filament is omitted. (B) Schematic diagram of the assay. The template dsDNA (70 bp) is labeled with Cy5 at the 17th base (from left) of the complementary strand. Cy3 is located at the ssDNA-dsDNA junction. (C) Anticipated slow changes of the fluorescence signals according to the model in A. (D-F) Typical time traces of observed fluorescence signals and the corresponding FRET.

Figure 2 (color in print) MT-based strand-exchange assay. (A) Schematic diagram of the assay. A dsDNA template (hairpin, $270 \mathrm{bp}$ ) is tethered to a glass slide and a magnetic bead with two DNA handles. The first 90 bp of the dsDNA template (colored olive and pink) are homologous to the 90-nt RecA-coated ssDNA (blue). Each opened base pair of the dsDNA template results in an increase in a DNA extension by $0.51+0.31=0.82 \mathrm{~nm}$ at $\sim 7 \mathrm{pN}$, where the first value is the mean length of 1-nt RecA-coated ssDNA and the second is that of 1-nt displaced ssDNA. (B) An MT trace for strand exchange with 100\% homology. (C) Statistical distribution of step sizes with 100\% homology. (D) An MT trace for strand exchange with $20 \%$ mismatches. 
Figure 3 (color in print) FRET-based assay for heteroduplex expansion in the presence of mismatches. (A) After strand exchange of the 30-bp homologous section, the rest of the dsDNA template attempts to incorporate into the complex for further strand expansion. Inset, The primary binding site and the secondary binding site of the RecA filament for DNA are separated by $\sim 2.5 \mathrm{~nm}$. (B-C) Typical FRET traces and histograms with $52 \%$ and 74\% mismatches. (D-E) The corresponding statistical distributions of the efficiencies of FRET bursts.

Figure 4 (color in print) Initiation of strand exchange measured by MT. (A) A typical MT trace with poly(T). (B) Statistical distribution of the initial alignment lengths. (C) Size distribution of the first steps in strand exchange without mismatch (MT assay in Fig. 2). (D) Free energy reductions associated with single-triplet additions to the heteroduplex according to the literature[18]. We propose that the heteroduplex does not expand in uniform 3-nt steps (Pathway 1, model in Fig. 1A) but may stride over several base triplets in a single step (Pathway 2). 


\section{Figures}

Figure 1 (color in print)

A
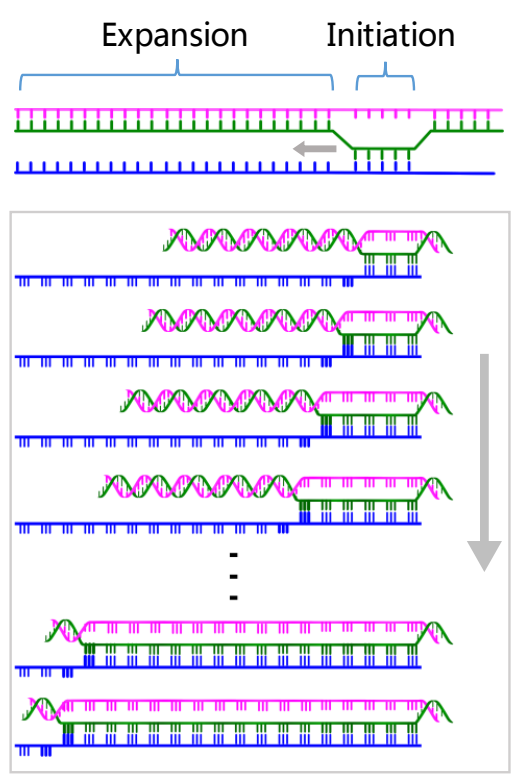

B
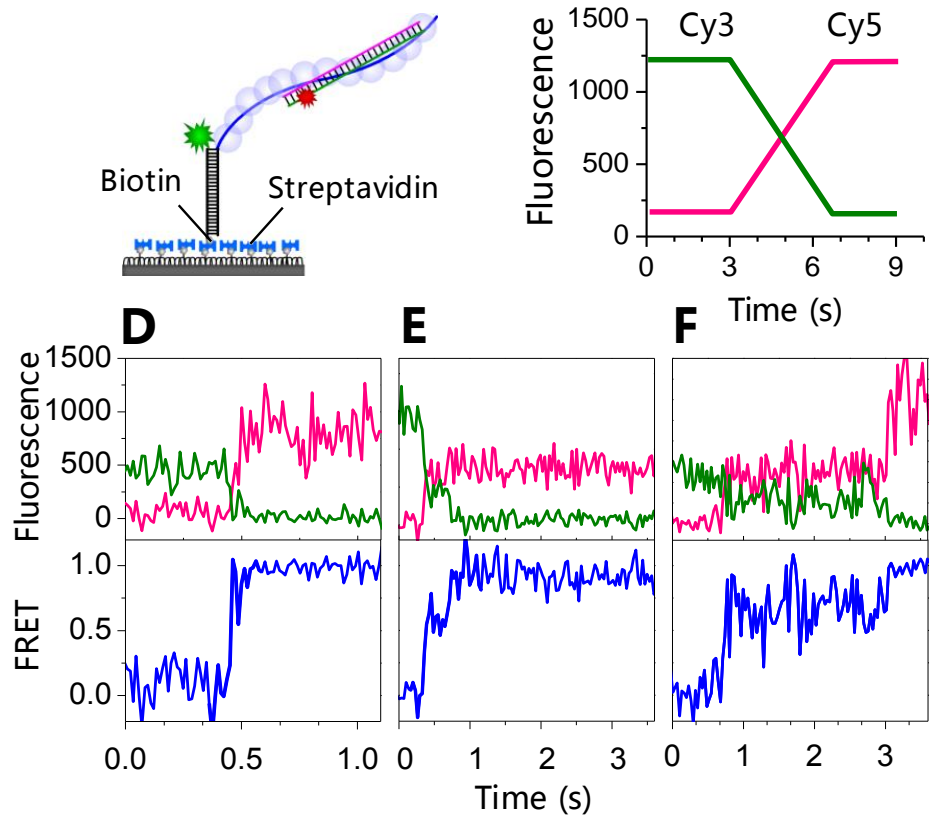
Figure 2 (color in print)

A

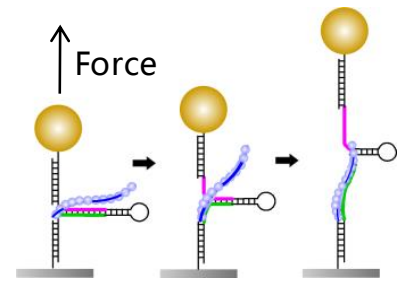

C

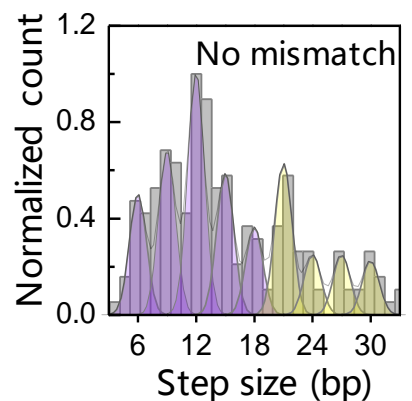

B
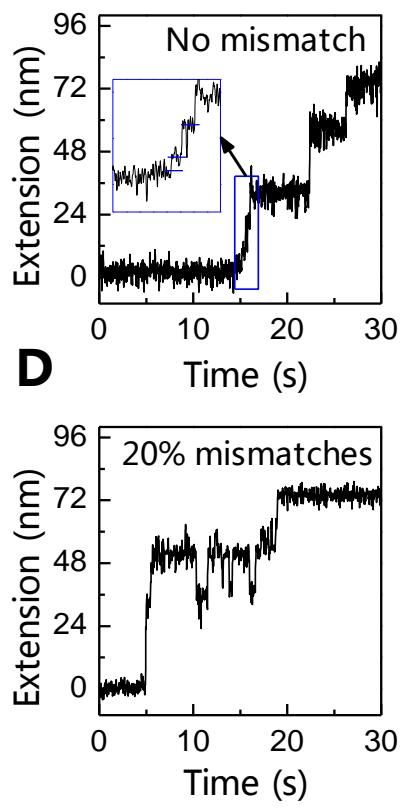
Figure 3 (color in print)
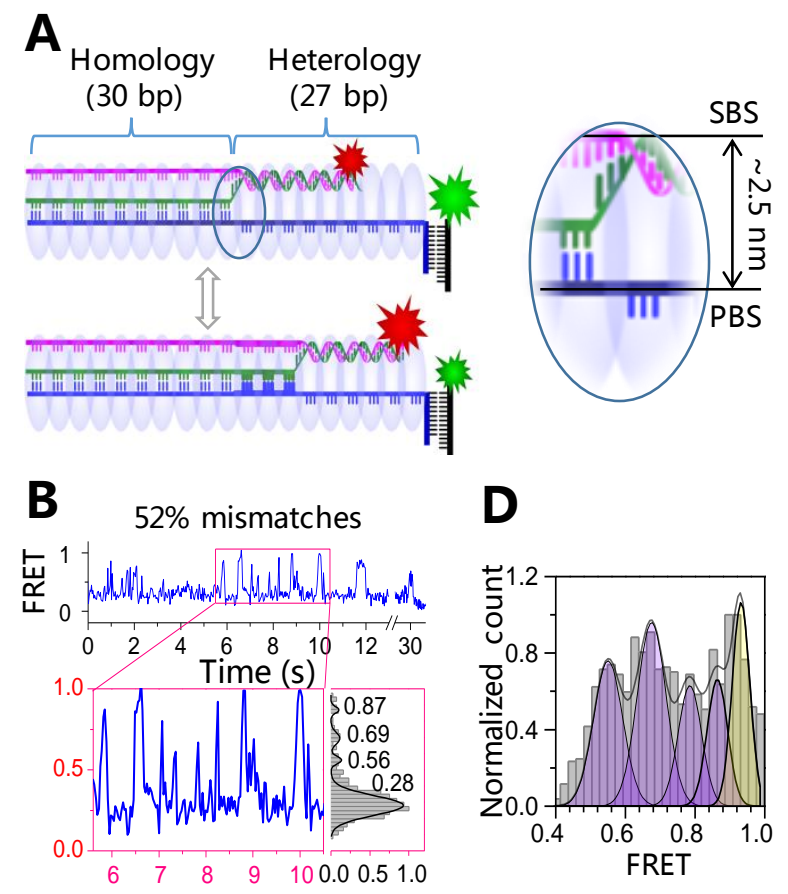

D
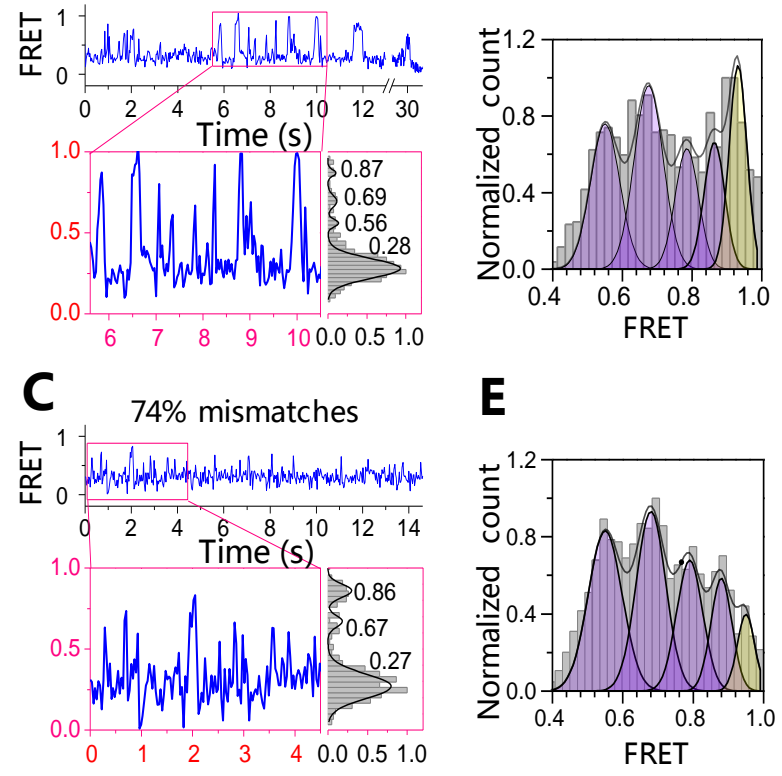

E

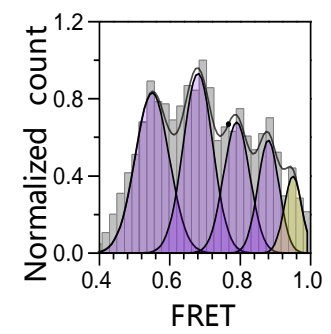


Figure 4 (color in print)
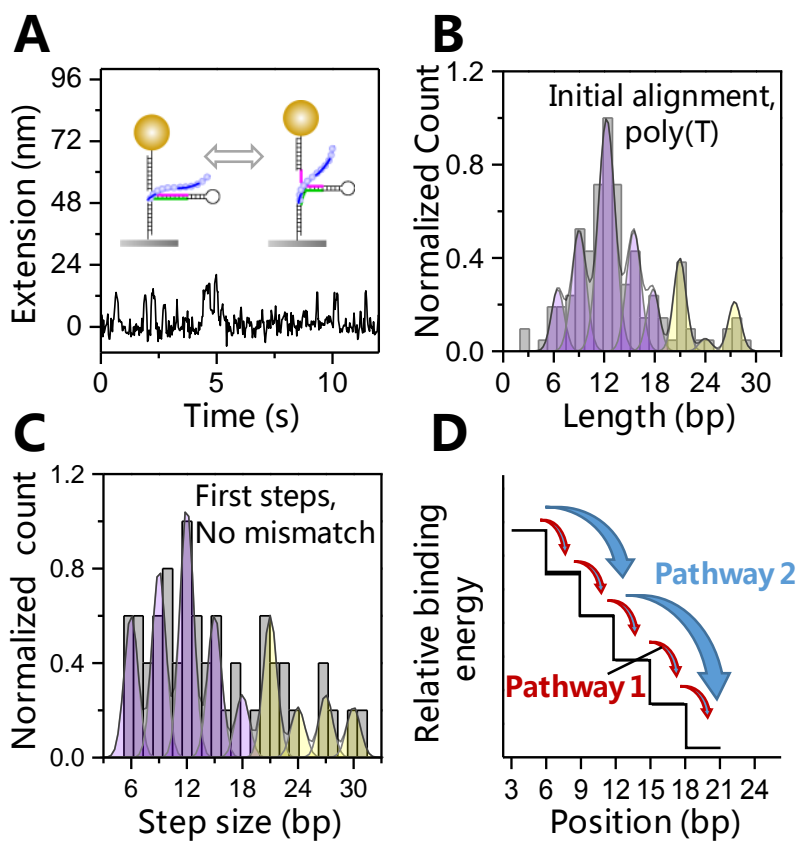